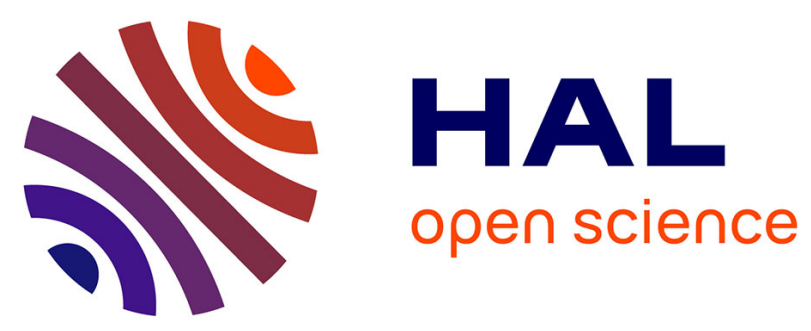

\title{
Une géométrie critique des lasers contenant des effets de lentille. II: Commentaire à " sur la longueur critique d'un laser " par G. Stephan
}

\author{
A. Le Floch, J.M. Lenormand, R. Le Naour, J.P. Taché
}

\section{To cite this version:}

A. Le Floch, J.M. Lenormand, R. Le Naour, J.P. Taché. Une géométrie critique des lasers contenant des effets de lentille. II: Commentaire à " sur la longueur critique d'un laser " par G. Stephan. Journal de Physique Lettres, 1984, 45 (7), pp.291-294. 10.1051/jphyslet:01984004507029100 . jpa-00232345

\section{HAL Id: jpa-00232345 https://hal.science/jpa-00232345}

Submitted on 1 Jan 1984

HAL is a multi-disciplinary open access archive for the deposit and dissemination of scientific research documents, whether they are published or not. The documents may come from teaching and research institutions in France or abroad, or from public or private research centers.
L'archive ouverte pluridisciplinaire HAL, est destinée au dépôt et à la diffusion de documents scientifiques de niveau recherche, publiés ou non, émanant des établissements d'enseignement et de recherche français ou étrangers, des laboratoires publics ou privés. 


\title{
LE JOURNAL DE PHYSIQUE-LETTRES
}

J. Physique Lett. 45 (1984) L-291 - L-294

1er AVRIL 1984, PAGE L-291

Classification

Physics Abstracts

$42.55 \mathrm{~F}-42.60 \mathrm{H}-42.60 \mathrm{D}$

\section{Une géométrie critique des lasers contenant des effets de lentille. II Commentaire à « sur la longueur critique d'un laser » par G. Stephan}

\author{
A. Le Floch $\left(^{*}\right)$, J. M. Lenormand $\left({ }^{*}\right)$, R. Le Naour $\left({ }^{* *}\right)$ et J. P. Taché $\left({ }^{*}\right)$ \\ (*) Laboratoire d'Electronique Quantique, Université de Rennes, F-35042 Rennes, France \\ $\left({ }^{* *}\right)$ Laboratoire d'Electronique Quantique, Université de Nantes, F-44072 Nantes, France
}

(Reçu le 20 juin 1983, révisé le 2 janvier 1984, accepté le 3 février 1984)

\begin{abstract}
Résumé. - Nous donnons le résultat d'une expérience qui montre que la géométrie critique, prévue par le formalisme de la matrice $A B C D$, existe lorsque le milieu remplit la cavité. Le concept de longueur critique n'exige pas l'hypothèse du champ moyen.
\end{abstract}

\begin{abstract}
We give the result of an experiment which shows that the critical length, predicted in the frame of the $A B C D$ matrix formalism, exists when the active medium fills the cavity. The critical length concept does not require the mean-field approximation hypothesis.
\end{abstract}

Suite à notre article intitulé " A critical geometry for lasers with internal lenslike effects », publié au J. Physique Lett. [1], un commentaire intitulé « Sur la longueur critique d'un laser » a été publié dans ce même journal [2]. De ce commentaire, retenons les trois principales conclusions :

i) "Sur le plan expérimental, nous donnons le résultat d'une expérience qui montre que le changement de dissymétrie n'existe pas quand le milieu remplit toute la cavité. Nous donnons ensuite une interprétation qui tient compte d'une nouvelle théorie de la forme de raie dans les lasers à gaz », page L-362.

ii) " Notre conclusion essentielle est donc que le concept de longueur critique est lié à l'hypothèse du champ moyen ", page L-363.

iii) «La théorie ab initio que nous avons faite permet de calculer ces phénomènes, contrairement à la théorie ad hoc des matrices $A B C D$ utilisées en général sans justification suffisante ", page L-368.

Nous proposons de montrer que ces conclusions ne sont pas fondées en indiquant la nécessité, d'une part, de préserver le caractère Gaussien du faisceau et, d'autre part, de tenir compte des 
variations importantes de la taille du faisceau d'une extrémité à l'autre de la cavité, pour réaliser la géométrie deux fois critique [3] par exemple.

Tout d'abord, sur le plan expérimental, nous apportons la preuve que, contrairement au résultat obtenu par l'auteur de [2], l'inversion de dissymétrie existe bien pour une cavité « quasi-pleine » (page L-363) comme celle qu'il a choisie. Cependant, pour obtenir ce résultat, il est essentiel de conserver le caractère Gaussien des faisceaux, ce qui constitue l'hypothèse fondamentale. Ceci implique de choisir un diamètre $2 r$ du diaphragme utilisé adapté au diamètre $2 w$ du mode. Une valeur de $r$ supérieure ou égale à $1,1 w$ est indispensable pour les raies à fort gain [4]. La figure 1 montre le résultat d'une expérience que nous avons faite avec un laser de mêmes caractéristiques que celui de G. Stephan [2] mais avec un diaphragme suffisamment grand $(r \simeq 1,2 w)$. La présence de fenêtres de Brewster aux extrémités du tube laser ne permet pas un remplissage total de la cavité mais les variations de taille de mode dans la cuve restent importantes, excluant l'hypothèse du champ moyen. Nous constatons que l'intensité de sortie du laser est la plus grande du côté des hautes fréquences alors que pour une petite longueur de cavité elle l'est du côté des basses fréquences [5] (dissymétrie « standard "). Ainsi, l'inversion de dissymétrie et la géométrie critique existent bien quand le milieu " remplit " la cavité. Par contre, si $r$ est choisi trop faible, par exemple $r=0,9 w$ comme le fait, dans son expérience, l'auteur de la référence [2], la déformation du faisceau Gaussien est importante $[4,6]$ (troncature et pertes de l'ordre de $20 \%$ ). Cette perte du caractère Gaussien des faisceaux, associée à un effet de focalisation supplémentaire non négligeable dû à la trop petite taille du diaphragme [7], masque alors l'inversion de dissymétrie attendue. Signalons de plus que, pour son analyse théorique, G. Stephan [2] utilise cependant un diaphragme de taille correcte $r=1,3 w$ (Fig. 2, page L-365).

Quant à la seconde conclusion de la référence [2], l'existence même de la géométrie critique avec une cuve longue, confirmée par notre figure 1 , montre que le concept de longueur critique ne

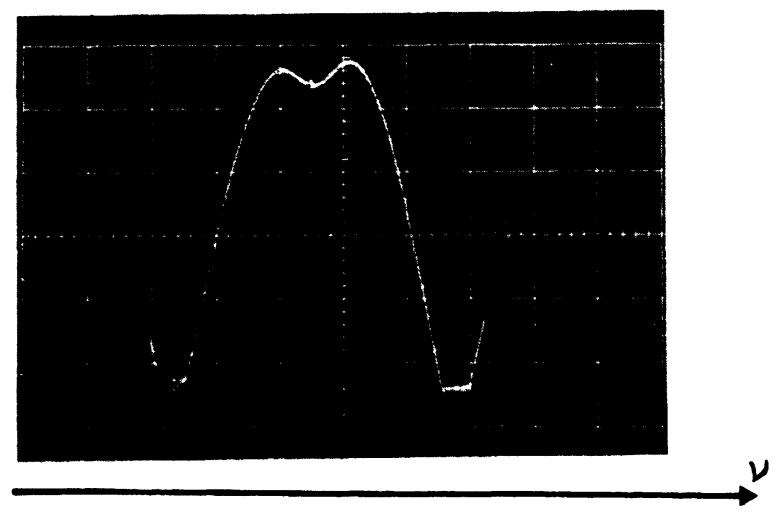

Fig. 1. - Courbe expérimentale représentant l'intensité de sortie du laser à 3,39 $\mu \mathrm{m}$ en fonction de la fréquence. La cavité, de longueur $57 \mathrm{~cm}$, est constituée d'un miroir plan et d'un miroir concave de rayon de courbure $60 \mathrm{~cm}$. Le diamètre du tube est de $8 \mathrm{~mm}$, sa longueur de $52 \mathrm{~cm}$. Le courant de décharge est de $10 \mathrm{~mA}$, la pression totale est de 0,5 torr $\left(\mathrm{Ne}^{20} / \mathrm{He}^{3} \simeq 1 / 5\right)$. Le diamètre du diaphragme, placé du côté du miroir concave, est de 3,9 mm $(2 r \simeq 2 \times 1,2 w)$. L'inversion de dissymétrie, par rapport à la dissymétrie observée en cavité courte $\left(d<d_{\mathrm{c}}\right)$ existe : le maximum est du côté hautes fréquences.

[Experimental output intensity of the $3.39 \mu \mathrm{m}$ laser versus the frequency. The cavity with a plane and a concave mirror $(60 \mathrm{~cm}$ curvature radius) is $57 \mathrm{~cm}$ long. The inner tube diameter is $8 \mathrm{~mm}$, its length $52 \mathrm{~cm}$. The current discharge is $10 \mathrm{~mA}$ for a total pressure of 0.5 torr $\left(\mathrm{Ne}^{20} / \mathrm{He}^{3} \simeq 1 / 5\right)$. The concave mirror aperture diameter is $3.9 \mathrm{~mm}(2 r \simeq 2 \times 1.2 \mathrm{w})$. The inversion of the standard asymmetry exists : the maximum of intensity is on the high frequency side.] 
résulte pas de l'hypothèse du champ moyen, hypothèse que nous n'avons d'ailleurs pas retenue dans ce cas. En effet, dans cette expérience, cette hypothèse n'est pas satisfaite car les variations de taille de mode sont importantes (voisines de 4,5) tout au long du laser et, de plus, l'intensité des deux ondes progressives à l'intérieur du laser varie notablement (transmission de $36 \%$ du miroir concave).

La théorie $a b$ initio [8] récemment développée par G. Stephan confirme, selon lui, son résultat expérimental. Elle n'a donc pas prévu l'existence d'une géométrie critique pour la cavité « quasipleine » étudiée. Cela n'est pas surprenant puisque cette théorie, dont la référence [2] était une première vérification, abandonne l'hypothèse du champ moyen dont résulte, selon l'auteur, le concept de longueur critique. Par contre, le formalisme de la matrice $A B C D$ permet de tenir compte des variations importantes des caractéristiques des faisceaux dans les cavités et de déterminer dans chaque cas, quand elle existe, la géométrie critique correspondante [3,9]. Nous avons ainsi étudié les variations de la longueur critique d'un laser en fonction de la position de la cuve amplificatrice dans le résonateur et proposé la géométrie doublement critique pour une cavité contenant un milieu absorbant et un milieu amplificateur [3]. Par ajustement de la position de la cuve absorbante, nous avons réalisé un pic symétrique sur fond plat, ce dernier étant lui-même obtenu par ajustement de la longueur, de la position et de l'excitation des deux cuves amplificatrices aux extrémités de la cavité.

L'effet de lentille dû à l'inhomogénéité transversale de population, étudié par Freed et Haus [10] notamment, peut intervenir dans les résultats expérimentaux. Il est à remarquer que, dans le cas étudié, comme l'indique la référence [2] (page L-367), il augmente l'intensité du côté des basses fréquences et, par conséquent, ne fait que masquer la géométrie critique due à la saturation. Cet effet de lentille de population ne peut d'ailleurs changer de signe en fonction du courant [11, 12], contrairement à l'hypothèse faite dans la théorie $a b$ initio [8]. En effet, pour un laser He-Ne à $3,39 \mu \mathrm{m}$, nous avons vérifié que la distribution radiale de gain ne présente pas de trou au centre, contrairement à ce qui existe pour la raie $3,51 \mu \mathrm{m}$ du xénon [13]. Nous pouvons également signaler que l'effet de lentille de population peut toujours être réduit en choisissant un tube de diamètre convenable [14] et n'existe pas, de toute façon, dans les milieux absorbants du type $\mathrm{CH}_{4}$ utilisés en métrologie [15] où le seul effet de lentille dû à la saturation permet de réaliser la géométrie critique.

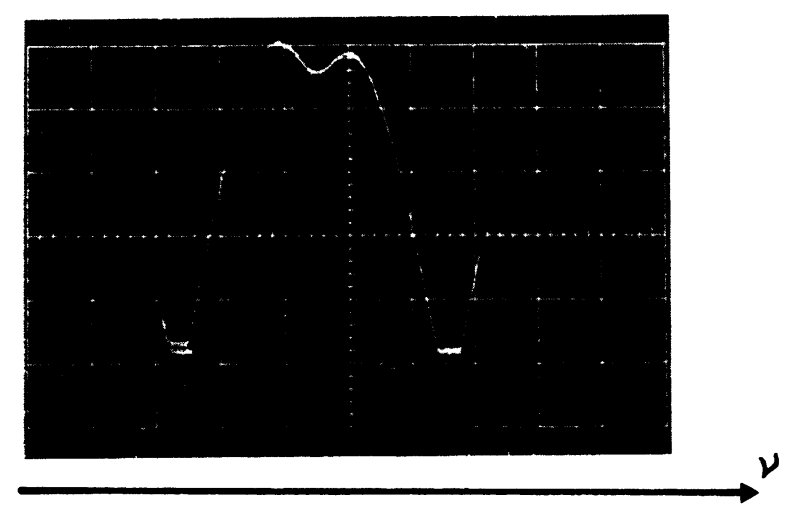

Fig. 2. - Même expérience que dans la figure 1 mais le diamètre du diaphragme est de $3 \mathrm{~mm}(2 r \simeq 2 \times$ $0,9 w$ ). L'inversion de dissymétrie n'existe plus : le maximum est du côté basses fréquences.

[Same experiment as in figure 1, but with an aperture diameter of $3 \mathrm{~mm}(2 r \simeq 2 \times 0.9 w)$. The inversion of asymmetry no longer exists : the maximum intensity remains on the low frequency side.] 
Nous constatons donc que, lorsque le milieu "remplit » la cavité, l'expérience réalisée par l'auteur de la référence [2] et confirmée par sa théorie de perturbation ab initio n'a pas permis de mettre en évidence l'inversion de dissymétrie. Par contre, la même expérience effectuée avec un faisceau Gaussien montre clairement l'existence de cette inversion.

\section{Bibliographie}

[1] Le Floch, A., Lenormand, J. M., Le Naour, R., TAChé, J. P., J. Physique Lett. 43 (1982) L-493.

[2] StÉPhan, G., J. Physique Lett: 44 (1983) L-361.

[3] Le Floch, A., Lenormand, J. M., Le NaOuR, R., Colloque D.R.E.T. des 25 et 26 mai 1982 et Revue du Céthédec, 3e trimestre 1983.

[4] Dembowski, J. et Weber, H., Opt. Commun. 42 (1982) 133.

[5] Le Floch, A., Le Naour, R., Lenormand, J. M., Rapport A.T.P. C.N.R.S. (17.11.81) et Colloque " Physique des Etats Excités ", Caen (1.02.82).

[6] Sifgman, A. E., An Introduction to Lasers and Masers (McGraw-Hill, New-York) 1971, p. 335.

[7] Otis, G., Lachambre, J. L., Lavigne, P., Appl. Opt. 18 (1979) 875.

[8] StÉPhan, G. et Trumper, M., Phys. Rev. A 28 (1983) 2344.

[9] Dans le cadre du formalisme de la matrice $A B C D$, la distance focale peut être obtenue en intégrant l'équation de propagation paraxiale dans un milieu présentant un effet de lentille. Une intégration numérique simple permet également de décrire l'évolution de la géométrie critique en fonction de la position, de la longueur, de l'excitation de la (ou des) cuve(s) et des variations d'intensité le long du laser par exemple.

[10] Freed, C. et Haus, H. A., I.E.E.E. J. Quantum Electron. QE-9 (1973) 219.

[11] Le Floch, A., Lenormand, J. M., Le NaOur, R., Ropars, G., Rapport D.R.E.T., 81-153.

[12] Mazanko, I. P., Molchanov, M. I., Ogurov, N. D. D., Sviridov, M. V., Opt. Spectrosc. (U.R.S.S.) 30 (1971) 495.

[13] Wolff, P. A., Abraham, N. B., Smith, S. R., I.E.E.E. J. Quantum Electron. QE-13 (1977) 400.

[14] Le Floch, A., Le Naour, R., Lenormand, J. M., TaChÉ, J. P., Phys. Rev. Lett. 45 (1980) 544.

[15] Le Floch, A., Lenormand, J. M., Le Naour, R., Brun, P., I.E.E.E. J. Quantum Electron. (octobre 1983). 